Fernanda Vilela Ferreira

\title{
Tecnologia industrial básica e inovação nas micro, pequenas e médias empresas de base tecnológica
}

Dissertação de Mestrado

Dissertação apresentada como requisito parcial para obtenção do grau de Mestre pelo Programa de PósGraduação em Metrologia. Área de Concentração: Negócios, Inovação e Metrologia. PUC-Rio.

Orientador: Prof. Antonio José Junqueira Botelho Co-orientadores: Prof. Maurício Nogueira Frota Prof ${ }^{a}$ Maria Fatima Ludovico de Almeida 


\title{
Fernanda Vilela Ferreira
}

\section{Tecnologia industrial básica e inovação nas micro, pequenas e médias empresas de base tecnológica}

\begin{abstract}
Dissertação apresentada como requisito parcial para obtenção do grau de Mestre pelo Programa de PósGraduação em Metrologia do Centro Técnico Científico da PUC-Rio.
\end{abstract}

Prof. Antonio José Junqueira Botelho

Orientador

Programa de Pós-Gradução em Metrologia PósMQI - PUC-Rio

Prof. Maurício Nogueira Frota Co-orientador

Programa de Pós-Gradução em Metrologia PósMQI - PUC-Rio

Prof ${ }^{\mathrm{a}}$ Maria Fatima Ludovico de Almeida Co-orientadora

Programa de Pós-Gradução em Metrologia PósMQI - PUC-Rio

Prof ${ }^{a}$ Renata Lebre La Rovere Instituto de Economia - UFRJ

Prof. Alex da Silva Alves

Programa de Pós-Gradução em Metrologia

PósMQI - PUC-Rio

Prof. José Eugênio Leal

Coordenador Setorial de Pós-Graduação do Centro Técnico Científico - PUC-Rio

Rio de Janeiro, 30 de julho de 2009 
Todos os direitos reservados. É proibida a reprodução total ou parcial do trabalho sem autorização da universidade, do autor e dos orientadores.

\section{Fernanda Vilela Ferreira}

Graduou-se em Engenharia Metalúrgica pelo Instituto Militar de Engenharia - IME. Possui pós-graduação em Gerenciamento de Projetos pela FGV. Atualmente é responsável pela estruturação e coordenação do Núcleo de Inovação Tecnológica do IME.

Ficha Catalográfica

Ferreira, Fernanda Vilela

Tecnologia industrial básica e inovação nas micro, pequenas e médias empresas de base tecnológica/ Fernanda Vilela Ferreira; orientadores: José Antonio Junqueira Botelho; Maurício Nogueira Frota; Maria Fatima Ludovico de Almeida - Rio de Janeiro: PUC, Programa de Pós-Graduação em Metrologia, 2009.

v., 117 f.: il. ; $29,7 \mathrm{~cm}$

1. Dissertação (mestrado) - Pontifícia Universidade Católica do Rio de Janeiro, Programa de Pós-Graduação em Metrologia

Inclui referências bibliográficas e anexo.

1. Metrologia - Teses. 2.Tecnologia Industrial Básica. 3. TIB. 4. Inovação tecnológica. 5. micro, pequenas e médias empresas. 6. MPMEs de base tecnológica. 7. empresas de base tecnológica. 8. EBT. 9. Brasil. 10. PINTEC. I. Botelho, Antonio José Junqueira. II. Frota, Maurício Nogueira. III. Maria Fatima Ludovico de Almeida. IV. Pontifícia Universidade Católica do Rio de Janeiro. Programa de PósGraduação em Metrologia. V. Título.

CDD: 389.1 


\section{Agradecimentos}

A toda minha família, em particular aos meus amados pais, Luiz Fernando e Neide, e irmã Flávia, por todo carinho, compreensão, incentivo, confiança. Obrigada por tudo!

Ao meu namorado, amigo e companheiro, Persio, por todo amor, apoio e compreensão.

Ao meu orientador Professor Antonio Botelho, pela atenção, motivação e conhecimentos transmitidos durante a condução desta dissertação.

Ao meu co-orientador, Professor Maurício Frota, pelo incentivo e críticas construtivas.

À minha co-orientadora, Professora Fátima Ludovico, meu anjo da guarda, a quem não tenho palavras para agradecer tamanho auxílio e dedicação.

Ao IME, pela oportunidade oferecida e pelo apoio no transcorrer de todo o curso.

Aos profissionais do IBGE pelo acesso aos dados da PINTEC, fundamentais para a realização da fase empírica da pesquisa.

Ao Professor Pimenta Bueno, pelos bons conselhos e insights fundamentais para o resultado desta pesquisa.

Aos funcionários do Programa de Pós-Graduação em Metrologia - Pós MQI, Márcia Ribeiro, Eline Girardi e Jaime Ticona pelo apoio qualificado durante o curso e a etapa de elaboração da dissertação.

A todos os meus colegas de turma que acompanharam de perto todo caminho percorrido durante o Mestrado, superando os desafios e comemorando comigo as vitórias conquistadas.

Aos meus grandes amigos e a todos aqueles que de alguma forma contribuíram para a realização deste trabalho. 


\section{Resumo}

Ferreira, Fernanda Vilela; Botelho, Antonio José Junqueira. Tecnologia industrial básica e inovação nas micro, pequenas e médias empresas de base tecnológica. Rio de Janeiro, 2009. 117p. Dissertação de Mestrado.Programa de Pós-Graduação em Metrologia. Área de concentração: Negócios, Inovação e Metrologia. Pontifícia Universidade Católica do Rio de Janeiro.

O objetivo da dissertação é contribuir para o avanço do conhecimento sobre a importância das funções da Tecnologia Industrial Básica (TIB) no ciclo de inovação das micro, pequenas e médias empresas (MPMEs) de base tecnológica no Brasil. Reconhecendo que essas empresas têm um papel fundamental no desenvolvimento econômico e social do país, a motivação da pesquisa é responder como elas percebem a importância das funções da TIB para o ciclo de inovação, na perspectiva de ressaltar os benefícios oriundos da consolidação da infraestrutura nacional de serviços tecnológicos de apoio a MPMEs de base tecnológica. A metodologia utilizada compreende: (i) revisão bibliográfica e documental para a elaboração do referencial teórico sobre os temas centrais da dissertação: MPMEs de base tecnológica; TIB; e inovação tecnológica; (ii) definição do objeto de estudo; da fonte primária de dados - Pesquisa de Inovação Tecnológica (PINTEC) do IBGE; e da grade de análise; (iii) solicitação ao IBGE de tabulações especiais da PINTEC; recebimento, formatação e análise dos dados; e elaboração e análise dos resultados; (iv) formulação das conclusões da pesquisa e de sugestões para a próxima PINTEC e estudos futuros. Destacam-se como resultados: (i) identificação de quatro itens da PINTEC diretamente associados às funções da TIB; (ii) análise da percepção das MPMEs brasileiras de base tecnológica sobre a relevância das funções da TIB para a inovação; (iii) análise comparativa dos padrões de respostas das MPMEs de base tecnológica inovadoras e não inovadoras. Como conclusão, tais resultados permitem afirmar que, em geral, essas empresas consideram as funções da TIB relevantes para suas atividades inovativas. A comparação entre as respostas das empresas inovadoras e não inovadoras reforça essa percepção: as inovadoras percebem melhor a importância de algumas funções da TIB do que as não inovadoras.

\section{Palavras-chave}

Tecnologia Industrial Básica; TIB; Inovação tecnológica; Micro, pequenas e médias empresas; MPMEs; MPMEs de base tecnológica; empresas de base tecnológica; EBT; Brasil; PINTEC. 


\section{Abstract}

Ferreira, Fernanda Vilela; Botelho, Antonio José Junqueira (Advisor). Infrastructural technologies and innovation in technology-based micro, small and medium size firms in Brazil. Rio de Janeiro, 2009. 117p. Master Dissertation - Programa de Pós-Graduação em Metrologia. Área de concentração: Negócios, Inovação e Metrologia. Pontifícia Universidade Católica do Rio de Janeiro.

The aim of this dissertation is to contribute to the understanding of some basic infrastructural technologies (TIB acronym in Portuguese) supporting innovation of technology-based micro, small and medium size enterprises (MSMEs) in Brazil. Given the critical role played by these firms in Brazil's social and economic development, the work seeks to answer how they perceive the importance of TIB in their innovation cycle. The methodology comprises: (i) a literature survey to elaborate a theoretical outline to characterize the core subjects of the dissertation: technology-based MSMEs; TIB; and technological innovation; (ii) definition of the object of study, of the primary data source - Technological Innovation Survey (Pesquisa de Inovação Tecnológica-PINTEC), of IBGE (Brazilian Statistical Office), and the analytic framework; (iii) to request IBGE to produce special tabulations; receipt, formatting and analysis of data; and analysis of results; and (iv) formulation of conclusions and suggestions for the next PINTEC survey and for future research. The main results can be summarized as follows: (i) identification of four PINTEC items associated with some TIB functions; (ii) identification and analysis of Brazilian technology-based MSMEs perceptions of the relevance of TIB functions for supporting innovation; and (iii) comparative analysis of patterns of response of innovative and non-innovative technology-based MSMEs. As conclusion, it can be said that, in general, these firms positively respond to TIB functions in innovation cycle. In particular, results show that innovative enterprises perceived better some TIB functions than the non-innovative ones.

\section{Keywords}

Infrastructural technologies; TIB; technologial innovation; micro, small and medium size enterprises; MSMEs; technology-based micro, small and medium size enterprises; new technology-based firm; NTBF; Brazil; PINTEC. 


\section{Sumário}

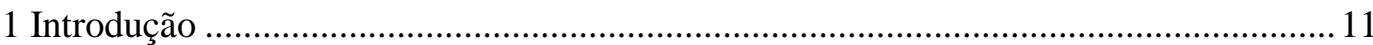

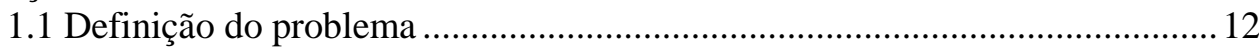

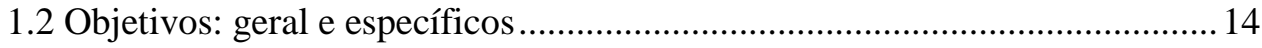

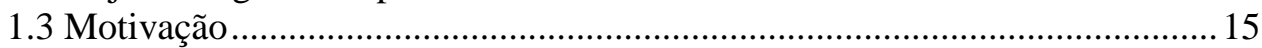

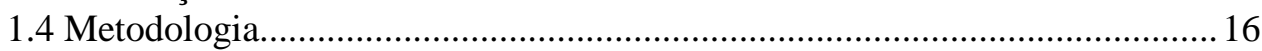

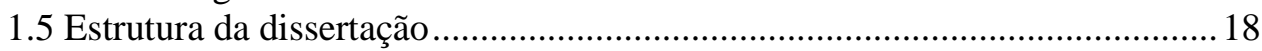

2 Micro, pequenas e médias empresas de base tecnológica ..............................................20

2.1 Micro, pequenas e médias empresas (MPMEs): conceitos ……..................22

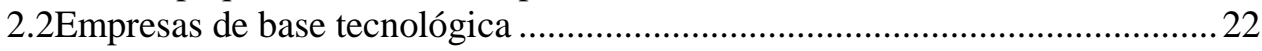

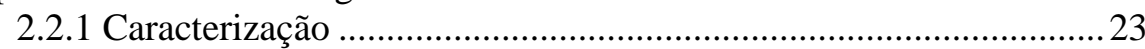

2.2.2 Natureza das MPMEs de base tecnológica no Brasil ....................22

2.3 Expressão econômica das MPMEs brasileiras de base tecnológica ................ 28

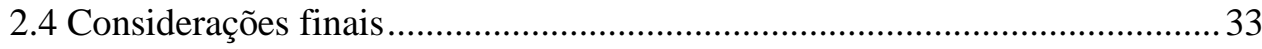

3 Inovação nas MPMEs de base tecnológica: conceitos, modelos e fontes de inovação ..34

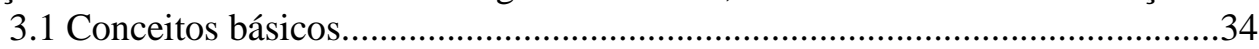

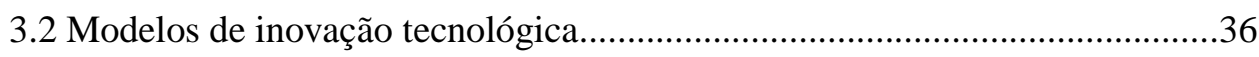

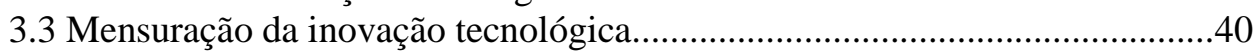

3.4 Fontes de inovação nas empresas.....................................................................

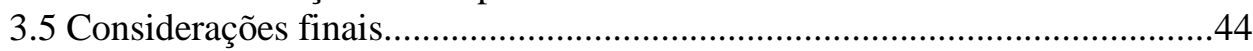

4 Tecnologia Industrial Básica como suporte à inovação das MPMEs de base

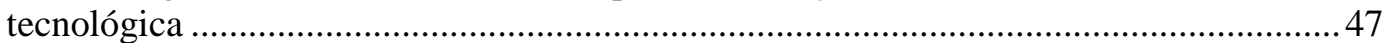

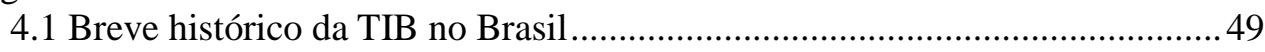

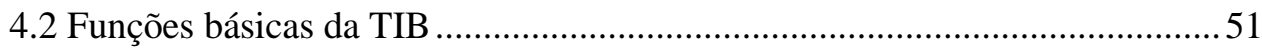

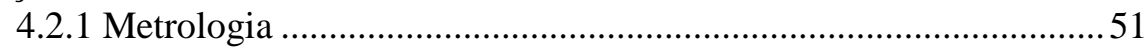

4.2.2 Normalização............................................................................... 55

4.2.3 Regulamentação técnica .............................................................59

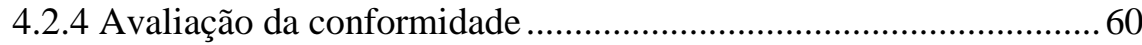

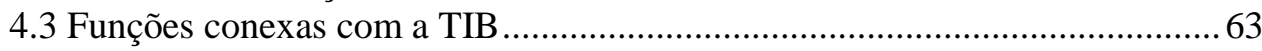

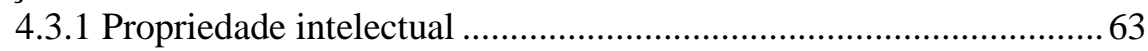

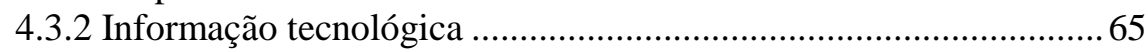

4.3.3 Tecnologias de gestão...................................................................66

4.4 Componentes da infraestrutura nacional de qualidade e inovação .................67

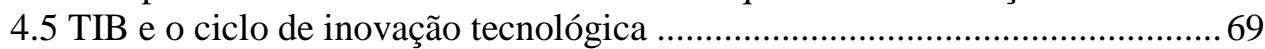

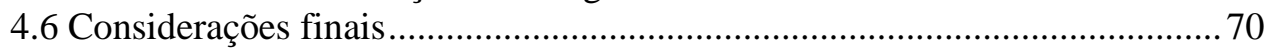

5 Inovação nas MPMEs brasileiras de base tecnológica..................................................... 72

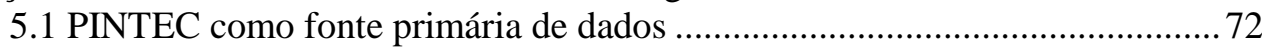

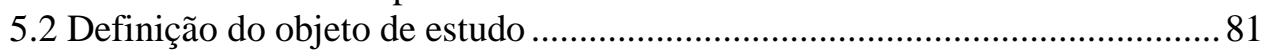

5.3 Perfil das MPMEs brasileiras de base tecnológica............................................ 84

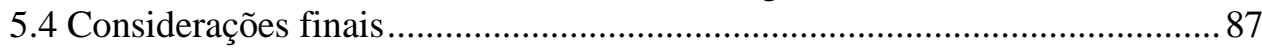

6 Tecnologia industrial básica e inovação nas MPMEs brasileiras de base tecnológica .. 88

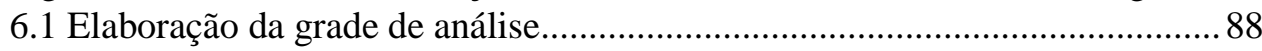

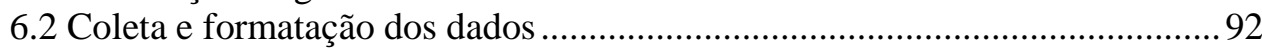

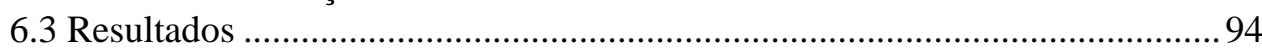

6.3.1 Dificuldade para se adequar a padrões, normas e

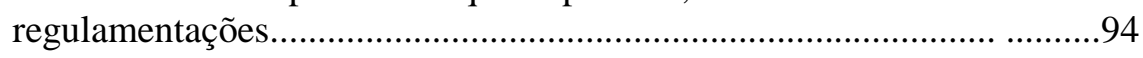

6.3.2 Escassez de serviços técnicos externos......................................... 98

6.3.3 Falta de informação sobre tecnologia.............................................99

6.3.4 Implementação de novos métodos, visando atender normas de certificação.................................................................................101

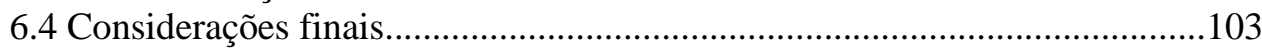

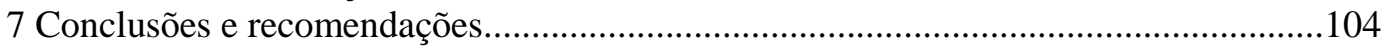

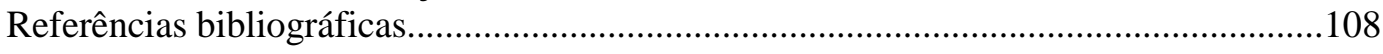




\section{Lista de Figuras}

Figura 1.1 - Definição do problema da pesquisa: questões a se investigar $\quad 13$

Figura 1.2 - Desenho da pesquisa, seus componentes e métodos 17

Figura 2.1 - Estrutura das exportações do Brasil (2003) e do mundo (2002) por 30 tipos e produtos classificados por intensidade tecnológica

Figura 2.2 - Participação quantitativa nas exportações por porte de empresa: 200632

Figura 2.3 - Participação percentual no valor exportado por porte de empresa: 200632

Figura 3.1 - Estrutura de mensuração da inovação tecnológica adotada no Manual de Oslo: foco nas MPMEs de base tecnológica

Figura 4.1 - Cadeia da TIB e infraestrutura tecnológica nacional

Figura 4.2 - As funções da TIB como suporte ao ciclo de inovação tecnológica

Figura 5.1 - Estrutura lógica do questionário da PINTEC

Figura 5.2 - Problemas e obstáculos apontados pelas empresas que implementaram inovações - Brasil - período 1998-2000 e período 2001-2003

Figura 5.3 - Problemas e obstáculos apontados pelas empresas que implementaram inovações, segundo atividades selecionadas de indústria e dos serviços - Brasil - período 2003-2005

Figura 6.1 - Representação da grade de análise da pesquisa

Figura 6.2 - Grau de importância atribuído pelas MPMEs de base tecnológica à dificuldade para se adequar a padrões, normas e regulamentações

Figura 6.3 - Resultados comparativos entre MPMEs de base tecnológica inovadoras e não inovadoras em relação ao grau de importância atribuído à dificuldade para se adequar a padrões, normas e regulamentações.

Figura 6.4 - Grau de importância atribuído pelas MPMEs de base tecnológica à escassez de serviços técnicos externos

Figura 6.5 - Resultados comparativos entre MPMEs de base tecnológica inovadoras e não inovadoras em relação ao grau de importância atribuído à escassez de serviços técnicos externos.

Figura 6.6 - Grau de importância atribuído pelas MPMEs de base tecnológica à falta de informação sobre tecnologia.

Figura 6.7 - Resultados comparativos entre MPMEs de base tecnológica inovadoras e não inovadoras em relação ao grau de importância atribuído à falta de informação sobre tecnologia

Figura 6.8 - Resultados comparativos entre MPMEs de base tecnológica inovadoras e não inovadoras em relação à implementação de novos métodos visando atender normas de certificação. 


\section{Lista de Tabelas}

Tabela 5.1 - Percentual das empresas inovadoras que consideraram como alto o grau de importância das atividades inovativas

Tabela 5.2 - MPMEs de base tecnológica identificadas no universo das empresas participantes da PINTEC

Tabela 5.3 - Taxa de inovação por porte de empresa

Tabela 5.4 - Participação percentual do número de empresas industriais que implementaram inovações, segundo faixas de pessoal ocupado

Tabela 6.1 - Número de empresas de base tecnológica em relação ao grau de importância atribuído à dificuldade para se adequar a padrões, normas e regulamentações

Tabela 6.2 - Número de empresas de base tecnológica em relação ao grau de importância atribuído à escassez de serviços técnicos externos

Tabela 6.3 - Número de empresas de base tecnológica em relação ao grau de importância atribuído à falta de informação sobre tecnologia

Tabela 6.4 - Número de empresas de base tecnológica que implementaram novos métodos, visando atender normas de certificação

Tabela 6.5 - Percentual de MPMEs de base tecnológica que implementaram novos métodos visando atender normas de certificação 


\section{Lista de Quadros}

Quadro 2.1 - Exemplos de classificações de empresas industriais segundo o seu 21 porte

Quadro 2.2 - Base de definição IBGE/Sebrae 21

Quadro 2.3 - Identificação das empresas de base Tecnológica 24

Quadro 3.1 - Prioridades das políticas de inovação européias 42

Quadro 3.2 - Fontes de inovação nas empresas $\quad 44$

Quadro 3.3 - Correspondências entre fontes de inovação e atividades inovativas $\quad 45$

Quadro 4.1 - Trajetória do fomento à TIB por meio do PADCT 49

Quadro 5.1 - Classificação do porte das empresas por número de empregados 81

Quadro 6.1 - Descrição da grade de análise 90 